\title{
POLIBETÁNICA
}

Núm. 39 pp. 151-174. México, 2015

\section{DIVERSIDAD DE MAÍZ EN LA SIERRA SUR DE OAXACA, MÉXICO: CONOCIMIENTO Y MANEJO TRADICIONAL}

\section{MAIZE DIVERSITY IN THE SOUTHERN REGION OF OAXACA, MEXICO: TRADITIONAL KNOWLEDGE AND TRADITIONAL MANAGEMENT}

\author{
Beatriz Rendón-Aguilar ${ }^{1 *}$, Verónica Aguilar-Rojas ${ }^{1}$, \\ María del Consuelo Aragón- Martínez ${ }^{1}$, José Francisco Ávila-Castañeda ${ }^{1}$, \\ Luis Alberto Bernal-Ramírez', David Bravo-Avilez', Guadalupe Carrillo-Galván', \\ Amelia Cornejo-Romero ${ }^{1}$, Ernesto Delgadillo-Durán ${ }^{2}$, Gilberto Hernández-Cárdenas ${ }^{1}$, \\ Mireya Hernández-Hernández ${ }^{1}$, Alejandro López-Arriaga ${ }^{1}$, \\ José Miguel Sánchez-García ${ }^{1}$, Eric Vides-Borrell ${ }^{1}$, y Rafael Ortega-Packzca ${ }^{3}$ \\ ${ }^{*}$ Universidad Autónoma Metropolitana-Iztapalapa, Departamento de Biología. \\ Oficina: 015558046449; Fax 015558044688. \\ ${ }^{2}$ Universidad Nacional Autónoma de México, Facultad de Ciencias. \\ ${ }^{3}$ Universidad Autónoma Chapingo. Correo electrónico: bra@xanum.uam.mx
}

\section{RESUMEN}

El estado de Oaxaca representa un importante acervo de maíz en México. Estudios previos han indicado la presencia de muchas variedades locales y razas agronómicas en la Sierra Norte y Valles Centrales. La Sierra Sur ha sido poco estudiada a pesar de la presencia de comunidades indígenas zapotecas, las cuales cultivan maíz, entre otras especies, y han preservado las variedades locales. El presente estudio responde a las siguientes preguntas: ¿cuál es la diversidad de maíz en términos de variedades tradicionales y razas agronómicas en la región zapoteca de Los Loxicha?, ¿la diversidad de maíz sigue un patrón de distribución a lo largo de un gradiente altitudinal?, ¿cuáles prácticas promueven y mantienen esta diversidad? Para contestar estas preguntas se realizó un estudio en cinco municipios de la Sierra Sur de Oaxaca. Durante 2007 se aplicaron entrevistas estructuradas a 930 agricultores para describir el proceso de manejo de semilla. La caracterización morfológica de las razas agronómicas se basó en muestras de mazorcas obtenidas en 375 milpas, con 20 plantas por milpa. Se encontraron altos niveles de diversidad local de maíz, con 36 variedades tradicionales correspondientes a 10 razas agronómicas. Las razas exhibieron diferencias en términos de variación fenotípica, distribución altitudinal y algunas muestran adaptación local. Los agricultores practican algunas formas de manejo ancestral para la selección de mazorcas y semillas, manteniendo, así, ciertos niveles de diferenciación entre las variedades y las razas agronómicas. Sin embargo, al sembrar diferentes variedades dentro de la misma parcela, se promueve el flujo génico y la introgresión entre ellas, manteniendo y promoviendo la diversidad fenotípica y genética.

Palabras clave: Zea mays, Sierra Sur de Oaxaca, intervalo altitudinal, razas agro- 
nómicas, manejo tradicional, zapotecos, variedades tradicionales.

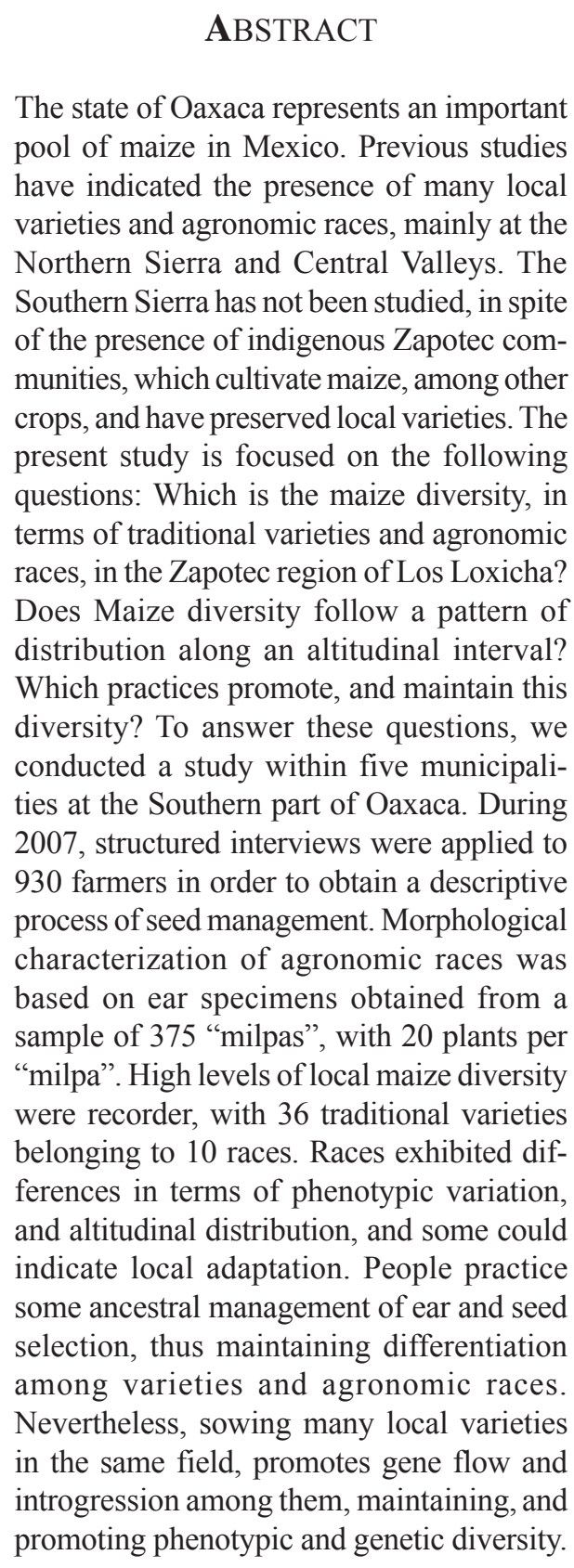

Key words: Zea mays, South Sierra of Oaxaca, altitudinal interval, agronomic races, traditional management, Zapotecs.

\section{INTRODUCCIÓN}

Una discusión reciente del origen del maíz en México ha reconsiderado al estado de Oaxaca como una de las áreas más antiguas e importantes de su domesticación y diversificación (Piperno \& Flannery, 2001; Smith, 2001; Matsuoka et al., 2002), particularmente en lo concerniente a las tierras altas de los Valles Centrales donde la domesticación del maíz parece haber ocurrido. Estas evidencias son complementadas con estudios que indican que en Oaxaca la diversidad genética del maíz es alta (Aragón-Cuevas et al., 2003; CONABIO, 2011).

Al presente, Oaxaca es considerado uno de los más importantes reservorios genéticos de maíz en México y en el mundo. El estado concentra 35 razas de maíz, lo que representa el $54 \%$ de las reportadas para todo el país (Aragón-Cuevas et al., 2003; Soleri et al., 2006). Casi el $90 \%$ del territorio agrícola del estado es cultivado con variedades tradicionales lo que representa, al mismo tiempo, el $10 \%$ del territorio mexicano (AragónCuevas et al., 2003; Soleri et al., 2006). La interacción entre las diversas condiciones ambientales encontradas en el territorio (e.g. orografía, humedad, suelos), así como el manejo y conocimiento tradicionales han generado variación morfológica que se expresa a través del ciclo de vida en los atributos fenotípicos de la planta (e.g. forma y tamaño de las mazorcas y plantas). También, en caracteres ecológicos como las variaciones en la respuesta a la presencia de enfermedades y plagas, características que en conjunto son reconocidas por los agricultores locales para 
la denominación de una variedad tradicional (también llamada: variedad criolla, nativa o local). Además, este proceso no tiene un final, ya que cada año el maíz se expone a variaciones en el ambiente y los agricultores van seleccionando la semilla que mejor responda a estas condiciones. Durante esta dinámica es posible que emerja una nueva variedad, la cual es claramente distinguible y apreciada por los agricultores (Hernández \& Ortega, 1973; Aguilar et al., 2003; Dyer \& Yúñez-Naude, 2003; Ortega-Paczka, 2003; Perales et al., 2003). También promueve un activo flujo génico incorporando nuevos alelos provenientes de variedades que son introducidas a las comunidades por diferentes procesos humanos (e.g. migración, comercialización, intercambio). En esta experimentación, la selección humana asegura los atributos de las variedades seleccionadas (Hernández \& Ortega, 1973; Aguilar et al., 2003; Ortega-Paczka, 2003). Cabe destacar además que, dentro de una raza agronómica están inmersas diversas variedades locales distribuidas en cada campo de cultivo.

La exploración y documentación de la diversidad morfológica y genética de maíz en el estado de Oaxaca se han centrado en las regiones norte y centro del estado (Smale et al., 1999; Aragón-Cuevas et al., 2000, 2002, 2006; Badstue et al., 2002; Soleri \& Smith, 2002; Dyer \& Yúñez-Naude, 2003; Pressoir \& Berthaud, 2004a, 2004b; Soleri et al., 2006). La Sierra Sur y Costa de Oaxaca han sido poco exploradas y se carece de estos registros.

El presente estudio se desarrolló en la región zapoteca de "Los Loxicha" localizada en la Sierra Sur de Oaxaca. Desde el punto de vista biológico y ecológico, es parte de la Región Terrestre Prioritaria (RTP) 129 (Arriaga et al., 2000) (fig. 1), la cual es considerada como una de las regiones más conservadas en cuanto a biodiversidad de todo el estado. Diversas comunidades zapotecas se han asentado ahí desde hace varias centenas de años y han transformado el ambiente a través de las actividades agrícolas principalmente, basadas en un uso y manejo tradicional de los recursos (Luna-José \& Rendón, 2008; Ventura-Aquino et al., 2008).

Algunos estudios no publicados sobre diversidad morfológica de maíz en esta región indican la existencia de al menos nueve variedades locales creciendo en el sistema de cultivo tradicional denominado "milpa". En el presente estudio se plantearon las siguientes preguntas: ¿cuál es la diversidad morfológica de maíz, en términos de variedades tradicionales y razas agronómicas en la región zapoteca de Los Loxicha?, ¿la diversidad morfológica del maíz sigue un patrón de distribución altitudinal? y ¿qué prácticas promueven y mantienen esta diversidad?

\section{MÉTODOS}

Área de estudio. La región de "Los Loxicha" se localiza en la Sierra Sur de Oaxaca, dentro de la región hidrológica conocida como "Copalita", entre los $16^{\circ} 00^{\prime} 30^{\prime}$ y $15^{\circ} 58^{\prime} 20^{\prime}$ " Latitud N y 96 $48^{\circ} 59^{\prime \prime}$ y $96^{\circ} 28^{\prime} 42^{\prime \prime}$ Longitud $\mathrm{O}$, en un intervalo altitudinal que va de 200 a 2050 m.s.n.m. (INEGI, 2000) (fig. 1, tabla 1). Debido a la diversidad biológica observada en el área, es considerada dentro de la RTP 129, conocida como Sierra Sur y Costa de Oaxaca (Arriaga et al., 2000) (fig. 1). Los zapotecos son el grupo étnico predominante en la región, pero la proporción de zapotecos-mestizos varía dentro de los municipios estudiados. 


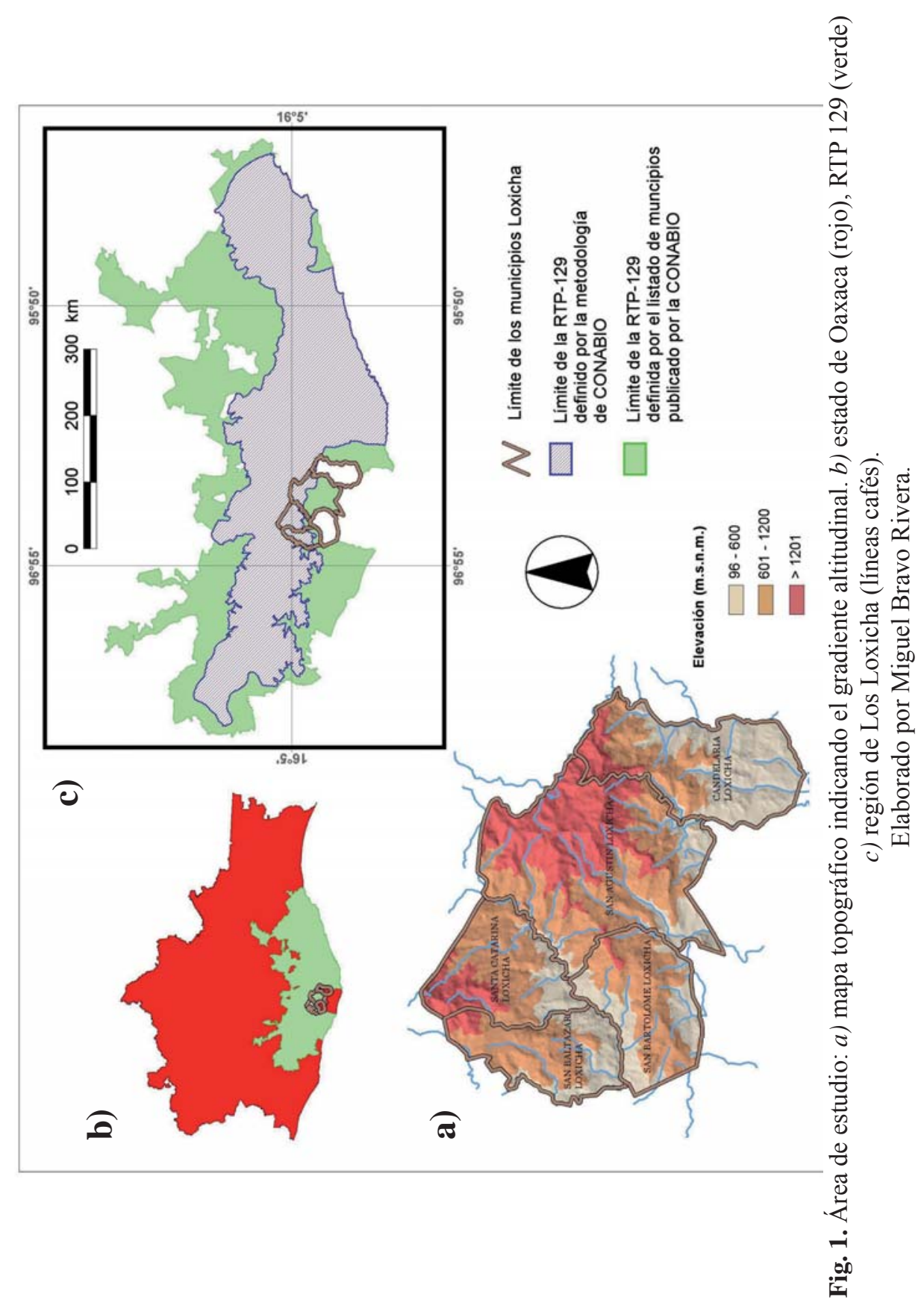


$\underline{\text { Rendón Aguilar, B. et al.: Diversidad de maíz en la Sierra Sur de Oaxaca, México: conocimiento y manejo tradicional }}$

Tabla 1. Características físicas y ecológicas de las localidades consideradas en el presente estudio. Las coordenadas geográficas y la altitud fueron registradas en la cabecera municipal.

\begin{tabular}{|c|c|c|c|c|}
\hline Municipio & Localidad & $\begin{array}{l}\text { Coordenadas } \\
\text { geográficas }\end{array}$ & $\begin{array}{c}\text { Altitud } \\
\text { (m.s.n.m.) }\end{array}$ & $\begin{array}{c}\text { Tipo de } \\
\text { vegetación }\end{array}$ \\
\hline \multirow[t]{4}{*}{ Candelaria Loxicha } & \multirow[b]{2}{*}{ Los Horcones } & $\begin{array}{l}15^{\circ} 48^{\prime} \text { y } 15^{\circ} 50^{\prime} \mathrm{N} \\
96^{\circ} 31^{\prime} \text { y } 96^{\circ} 37^{\prime} \mathrm{O}\end{array}$ & $144-376$ & $\mathrm{SBC}$ \\
\hline & & $\begin{array}{c}15^{\circ} 48^{\prime} \text { y } 15^{\circ} 56^{\prime} \mathrm{N} \\
96^{\circ} 34^{\prime} \mathrm{O}\end{array}$ & $128-419$ & SBC \\
\hline & Río Molino & $\begin{array}{c}15^{\circ} 56^{\prime} \mathrm{N} \\
96^{\circ} 30^{\prime} \text { y } 96^{\circ} 31^{\prime} \mathrm{O}\end{array}$ & $758-834$ & SMSp \\
\hline & $\begin{array}{c}\text { Santa María } \\
\text { Tepejipana }\end{array}$ & $\begin{array}{l}15^{\circ} 48^{\prime} \text { y } 16^{\circ} 01^{\prime} \mathrm{N} \\
96^{\circ} 33^{\prime} \text { y } 96^{\circ} 35^{\prime} \mathrm{O}\end{array}$ & $163-799$ & SBC-SMSp-BE \\
\hline \multirow[t]{5}{*}{ San Agustín Loxicha } & \multirow[b]{3}{*}{ San Francisco Loxicha } & $\begin{array}{l}15^{\circ} 48^{\prime} \text { y } 15^{\circ} 50^{\prime} \mathrm{N} \\
96^{\circ} 31^{\prime} \text { y } 96^{\circ} 37^{\prime} \mathrm{O}\end{array}$ & 1100 & BE-BPE-BP \\
\hline & & $\begin{array}{l}16^{\circ} 00^{\prime} \text { y } 16^{\circ} 01^{\prime} \mathrm{N} \\
96^{\circ} 34^{\prime} \text { y } 96^{\circ} 35^{\prime} \mathrm{O}\end{array}$ & $1804-2114$ & BE-BPE-BP \\
\hline & & $\begin{array}{l}15^{\circ} 52^{\prime} \text { y } 15^{\circ} 54^{\prime} \mathrm{N} \\
96^{\circ} 35^{\prime} \text { y } 96^{\circ} 41^{\prime} \mathrm{O}\end{array}$ & $300-517$ & SBC-SMSp-BMM \\
\hline & San Vicente Yogondoy & $\begin{array}{l}16^{\circ} 03^{\prime} \text { y } 16^{\circ} 05^{\prime} \mathrm{N} \\
96^{\circ} 35^{\prime} \text { y } 96^{\circ} 38^{\prime} \mathrm{O}\end{array}$ & $930-1583$ & BE-BPE-BP \\
\hline & $\begin{array}{c}\text { Santa María } \\
\text { Magdalena Loxicha }\end{array}$ & $\begin{array}{l}15^{\circ} 52^{\prime} \text { y } 15^{\circ} 54^{\prime} \mathrm{N} \\
96^{\circ} 40^{\prime} \text { y } 96^{\circ} 42^{\prime} \mathrm{O}\end{array}$ & $300-539$ & SBC \\
\hline \multirow[t]{3}{*}{ San Baltazar Loxicha } & \multirow[b]{2}{*}{ San Baltazar Loxicha } & $\begin{array}{l}15^{\circ} 57 \mathrm{~N} \\
96^{\circ} 49 \mathrm{O}\end{array}$ & 155 & SBC \\
\hline & & $\begin{array}{l}15^{\circ} 59^{\prime} \text { y } 16^{\circ} 04^{\prime} \mathrm{N} \\
96^{\circ} 48^{\prime} \text { y } 96^{\circ} 49^{\prime} \mathrm{O}\end{array}$ & $406-1500$ & SMSp-BE \\
\hline & Santa Martha Loxicha & $\begin{array}{c}16^{\circ} 00^{\prime} \mathrm{N} \\
96^{\circ} 43^{\prime} \text { y } 96^{\circ} 48^{\prime} \mathrm{O}\end{array}$ & $735-841$ & SMSp-BE-BP \\
\hline \multirow[t]{3}{*}{ San Bartolomé Loxicha } & San Bartolomé Loxicha & $\begin{array}{l}15^{\circ} 54^{\prime} \text { y } 15^{\circ} 59^{\prime} \mathrm{N} \\
96^{\circ} 41^{\prime} \text { y } 96^{\circ} 44^{\prime} \mathrm{O}\end{array}$ & $280-1293$ & $\begin{array}{c}\text { SBC-SMSp- } \\
\text { BMM-BE-BEP-BP }\end{array}$ \\
\hline & La Chinilla & $\begin{array}{c}15^{\circ} 55^{\prime} \mathrm{N} \\
96^{\circ} 47^{\prime} \text { y } 96^{\circ} 49^{\prime} \mathrm{O}\end{array}$ & $533-839$ & SBC-SMSp \\
\hline & El Tamarindo & $\begin{array}{l}15^{\circ} 53^{\prime} \text { y } 15^{\circ} 59^{\prime} \mathrm{N} \\
96^{\circ} 43^{\prime} \text { y } 96^{\circ} 44^{\prime} \mathrm{O}\end{array}$ & $443-774$ & SBC-SMSp \\
\hline Santa Catarina Loxicha & Santa Catarina Loxicha & $\begin{array}{l}15^{\circ} 55^{\prime} \text { y } 16^{\circ} 06^{\prime} \mathrm{N} \\
96^{\circ} 42^{\prime} \text { y } 96^{\circ} 48^{\prime} \mathrm{O}\end{array}$ & $500-1830$ & $\begin{array}{l}\text { SMSp-BE-BP- } \\
\text { BMM }\end{array}$ \\
\hline
\end{tabular}

Claves para el tipo de vegetación: $\mathrm{SBC}=$ selva baja caducifolia; $\mathrm{SMSp}=$ selva mediana subperennifolia; $\mathrm{BMM}=$ bosque mesófilo de montaña; $\mathrm{BPE}=$ bosque de pino-encino; $\mathrm{BE}=$ bosque de encino; $\mathrm{BP}=$ bosque de pino. 
San Agustín Loxicha presenta la mayor proporción de zapotecos, mientras que las comunidades restantes la población mestiza es mayor (INEGI, 2011).

Se seleccionaron los cinco municipios que conforman la región de Los Loxicha (San Agustín Loxicha, Candelaria Loxicha, San Bartolomé Loxicha, Santa Catarina Loxicha y San Baltazar Loxicha). La agricultura es la principal actividad económica, siendo el maíz y el café los cultivos básicos, los cuales se cultivan dentro de sistemas agrícolas tradicionales (milpa y café de sombra). Los efectos de la introducción de la tecnología durante los años 70 se observan en la aplicación de algunas prácticas agrícolas, como es el uso de pesticidas y fertilizantes inorgánicos, los cuales son empleados sin control o sin seguir un programa técnico.

Se realizó una estratificación de la zona de estudio con el objeto de determinar rangos de distribución de cada variedad tradicional y raza agronómica: zona 1, 0-600 m.s.n.m.; zona 2, 601 a 1200 m.s.n.m. y zona 3, más de $1200 \mathrm{~m}$ s.n.m. Dentro de cada municipio, se seleccionaron comunidades con al menos 300 habitantes (basado en datos del INEGI, 2000), obteniendo un total de 13 comunidades. El maíz es el cultivo más importante en esta región, y recibe respeto por parte de la gente. Por tal motivo, fue necesario presentar el proyecto a las autoridades locales de cada municipio, con el objeto de facilitar la colecta de mazorcas y granos.

Entrevistas. Entre julio y octubre del 2007 se aplicó una encuesta cerrada a 930 agricultores seleccionados de manera aleatoria en cada comunidad. El objetivo de esta encuesta fue entender las preferencias que los agricultores tienen sobre las variedades tra- dicionales que siembran, tiempo que tienen sembrando cada variedad, la procedencia de la semilla y los criterios que siguen para seleccionar las mazorcas y la semilla.

Se registraron los nombres locales asignados a cada variedad tradicional. La variedad tradicional se define como las variedades que se han seleccionado localmente, e incluye las variantes locales, razas geográficas, variedades mejoradas acriollizadas e híbridos interraciales que se han cultivado por varios años y que los agricultores conservan y siembran a partir de lotes de semillas (Brush \& Perales, 2007). Se utilizó el nombre de variedad tradicional cuando un fenotípico específico es reconocido y nombrado por los agricultores (Louette \& Smale, 2000; Perales et al., 2003).

Muestreo de mazorcas y semillas. Entre octubre de 2007 y febrero de 2008 se visitaron 375 milpas con el propósito de obtener muestras de mazorcas. Dentro de cada una, se eligieron aleatoriamente 20 plantas de maíz y una mazorca de cada planta fue recolectada. Se hicieron diversas mediciones: largo y ancho de la mazorca y el olote; número de hileras de grano; longitud, largo, ancho y espesor de 10 semillas/mazorca y volumen de 100 granos. Se obtuvieron datos adicionales como altura total de la planta y altura de la primera mazorca. Se registraron la altitud, las coordenadas geográficas y porcentaje de inclinación de la pendiente de cada milpa. Las mazorcas se etiquetaron y entregaron al Banco de Germoplasma de la Universidad Autónoma Chapingo. Estos datos se utilizaron para describir las diferentes razas, apoyándose en los descriptores previos (Wellhausen et al., 1952; Sánchez et al., 2000; Herrera-Cabrera et al., 2004; Pressoir \& Berthaud, 2004a, 2004b). 
Análisis de datos. Se analizó el número total de variedades tradicionales y de razas, su distribución dentro y entre comunidades y municipios, y a lo largo del gradiente altitudinal. Como se indicó previamente, se consideró a una variedad tradicional como aquel fenotipo que es reconocido por un grupo de agricultores. La distribución altitudinal de cada variedad tradicional fue determinada considerando la altitud menor y mayor donde las muestras fueron colectadas. Los datos de las mazorcas y las semillas fueron analizados con Análisis de Varianza de una sola vía, siendo la raza la variable independiente. Se aplicó una prueba de Tukey para determinar las diferencias entre las razas. Se aplicaron modelos de regresión lineal para determinar las posibles relaciones entre algunas características de la mazorca o la semilla y la altitud. El doctor Rafael Ortega-Paczka, de la Universidad Autónoma Chapingo, clasificó las mazorcas de cada agricultor, siguiendo los criterios de Wellhausen's et al. (1952).

\section{RESUlTADOS}

Variedades tradicionales de maíz. Se registraron 36 variedades tradicionales de maíz para la zona de estudio, tomando como base los fenotipos que la gente reconoce con el mismo nombre (tabla 2). Las variedades presentan una distribución a lo largo de un gradiente altitudinal. Algunas se localizan únicamente en las partes altas, arriba de los $1200 \mathrm{~m}$, como las variedades "blanco", "amarillo grande serrano" y "tablita" (fig. 2). El $40 \%$ se distribuyen en altitudes bajas y medias. Esta distribución es independiente de la frecuencia con que fue mencionada por los agricultores. Por ejemplo, la variedad "pinto" fue muy poco mencionada, pero se cultiva en las partes altas y bajas de la zona de estudio. Por el contario, la variedad "chaparro" tuvo una alta frecuencia de mención, pero se distribuye únicamente por debajo de los 600 m s.n.m. (fig. 2, tabla 2).

Las variedades con mayor frecuencia de mención fueron "tablita", "tempranero", "blanco" y "chaparrón", mientras "marteño", "negro", "pinto", "ndixh" y "maguellano" tuvieron muy baja frecuencia de mención (fig. 3). 19 variedades tradicionales (41\%), fueron mencionadas únicamente por una persona en solo dos comunidades: $36 \%$ en Santa Catarina Loxicha, y $21 \%$ en San Vicente Yogondoy, pero dentro de un amplio rango altitudinal. En relación a los municipios, San Agustín Loxicha y San Baltazar Loxicha tuvieron el mayor número de variedades únicas (fig. 4).

Algunas características agroecológicas y fenotípicas están relacionadas con la altitud. Por ejemplo, "chaparro", "enano" u "olotillo", se distribuyen entre los 240 y los 1200 m.s.n.m. Su ciclo de vida dura de tres a cuatro meses, crecen en áreas secas con pendientes ligeras en sitios con vegetación de selva baja caducifolia. En las partes medias $y$ altas las variedades tradicionales exhiben ciclos de vida más largos, entre cuatro y seis meses, y algunos incluso hasta más.

Es frecuente encontrar que algunas variedades tradicionales corresponden a diferentes razas agronómicas pero comparten características similares y, en consecuencia, reciben el mismo nombre local. Por ejemplo, la variedad tradicional "blanco" corresponde a las razas vandeño, mushito, tuxpeño, tepecintle y olotillo; "hueso amarillo" está presente en zapalote grande, mushito, tepecintle y olotillo. Sólo las va- 


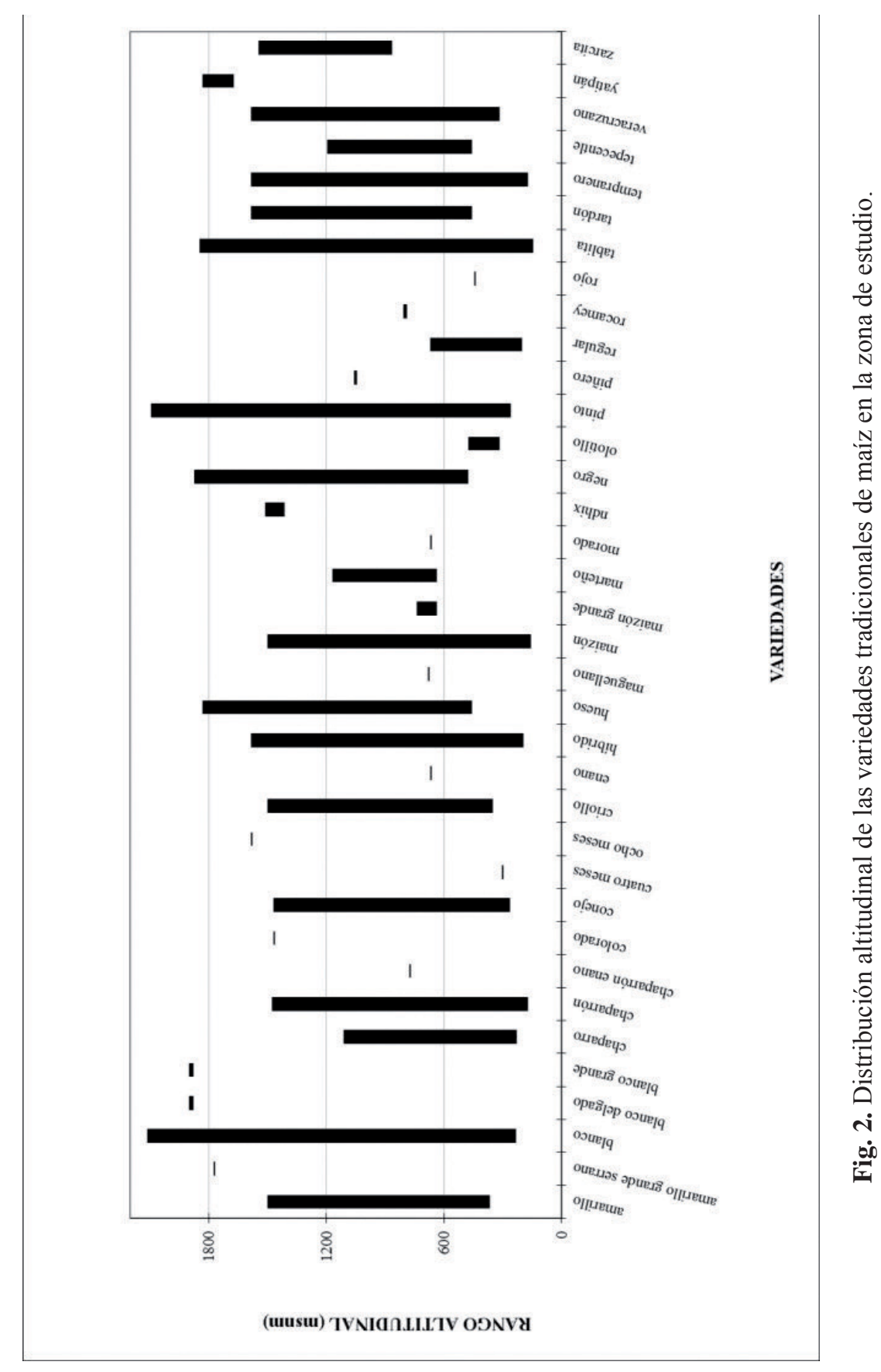


Rendón Aguilar, B. et al.: Diversidad de maíz en la Sierra Sur de Oaxaca, México: conocimiento y manejo tradicional

Tabla 2. Variedades tradicionales (VT) registradas en el sitio de estudio, Los Loxicha, Oaxaca.

\begin{tabular}{|c|c|c|}
\hline Nombre local & $\begin{array}{l}\text { Número de } \\
\text { menciones }\end{array}$ & $\begin{array}{c}\text { Intervalo } \\
\text { altitudinal }\end{array}$ \\
\hline amarillo & 36 & $366-1500$ \\
\hline amarillo grande serrano & 1 & $1773-1773$ \\
\hline blanco & 119 & $235-2114$ \\
\hline blanco delgado & 2 & $1881-1900$ \\
\hline blanco grande & 2 & $1881-1900$ \\
\hline chaparro & 33 & $229-1109$ \\
\hline chaparrón & 77 & $172-1476$ \\
\hline chaparrón enano & 3 & $774-774$ \\
\hline colorado & 2 & $1468-1468$ \\
\hline conejo & 13 & $264-1468$ \\
\hline cuatro meses & 1 & $300-300$ \\
\hline ocho meses & 1 & $1583-1583$ \\
\hline criollo & 10 & $353-1500$ \\
\hline enano & 2 & $668-668$ \\
\hline híbrido & 38 & $194-1583$ \\
\hline hueso (amarillo y blanco) & 127 & $459-1830$ \\
\hline maguellano & 1 & $680-680$ \\
\hline maizón (amarillo y blanco) & 153 & $155-1500$ \\
\hline maizón grande & 2 & $637-735$ \\
\hline marteño & 10 & $637-1167$ \\
\hline morado & 1 & $668-668$ \\
\hline ndhix & 2 & $1416-1511$ \\
\hline negro & 3 & $478-1871$ \\
\hline olotillo & 4 & $316-474$ \\
\hline pinto & 15 & $259-2094$ \\
\hline piñero & 2 & $1046-1057$ \\
\hline regular (blanco) & 16 & $201-668$ \\
\hline rocamey & 2 & $788-803$ \\
\hline rojo & 1 & $442-442$ \\
\hline tablita (amarillo y blanco) & 313 & $144-1845$ \\
\hline tardón & 3 & $459-1583$ \\
\hline tempranero (amarillo, blanco y colorado) & 73 & $173-1583$ \\
\hline tepecentle (amarillo, blanco, pinto) & 30 & $459-1192$ \\
\hline veracruzano & 5 & $316-1583$ \\
\hline yatipán & 2 & $1674-1830$ \\
\hline zarcita (amarillo y blanco) & 4 & $865-1546$ \\
\hline
\end{tabular}




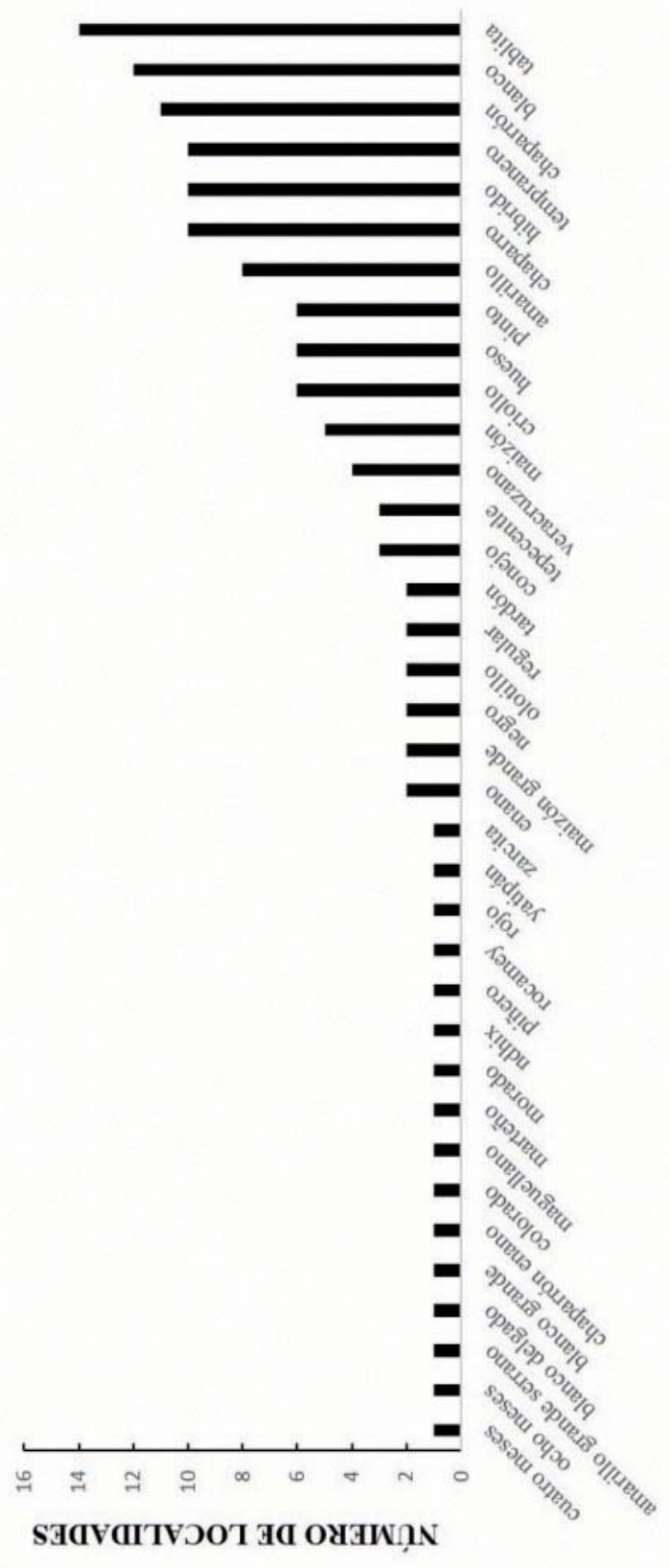




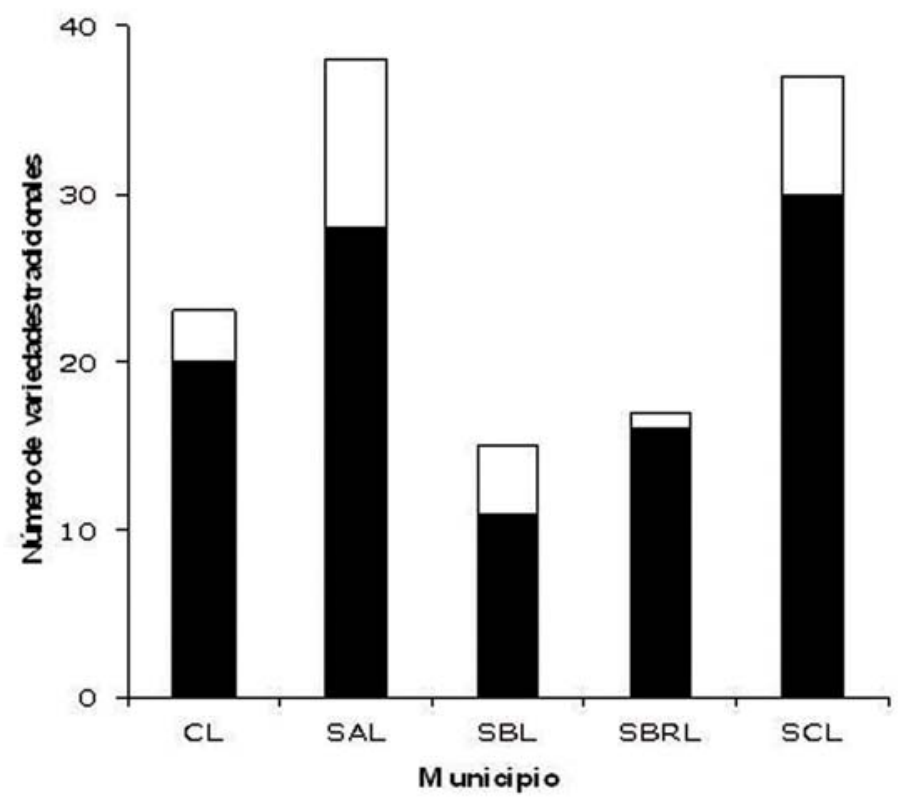

Fig. 4. Número de variedades tradicionales registradas en cada municipio (negro). Número de variedades únicas registradas (blanco).

$\mathrm{CL}=$ Candelaria Loxicha; $\mathrm{SAL}=$ San Agustín Loxicha; $\mathrm{SBL}=$ San Baltazar Loxicha; SBRL $=$ San Bartolomé Loxicha; SCL = Santa Catarina Loxicha.

riedades tradicionales "blanco grande" y "maizón" corresponden, respectivamente, a una sola raza: comiteco y tabloncillo (fig. 2). Al comparar con algunos estudios en diferentes estados de México, y aún en el estado de Oaxaca, la diversidad reportada en este estudio es alta en términos de las variedades tradicionales y razas mencionadas; estudios previos realizados en diferentes comunidades de los Valles Centrales de Oaxaca (Smale et al., 1999; Bellon, 2004; Bellon et al., 2006) y en la región Mixteca (Chávez-Servia et al., 2011; Soriano et al. 2011; Vera-Guzmán, 2012) reportan menor diversidad fenotípica.

\section{Clasificación de las variedades tradicionales en razas}

Las 36 variedades tradicionales fueron agrupadas en 10 razas agronómicas, de acuerdo a los criterios propuestos por Wellhausen $e t$ al. (1952) (tablas 2 y 3). Este registro representa el $20 \%$ de aquellas reportadas para todo el país. Según la revisión bibliográfica, la raza vandeño representó un nuevo registro para el estado (Aragón-Cuevas et al., 2006). El número de razas encontrado en el presente estudio fue mayor al registrado en estudios previos desarrollados incluso en áreas más extensas (Perales et al., 2003; 
Tabla 3. Número total de razas agronómicas registradas en el sitio de estudio. Se indican si corresponden a razas puras o que presentan introgresión.

\begin{tabular}{|c|c|c|}
\hline \multirow{2}{*}{ Raza agronómica } & \multicolumn{2}{|c|}{ Introgresión } \\
\cline { 2 - 3 } & Sin & Con \\
\hline comiteco & $\mathrm{x}$ & \\
\hline conejo & $\mathrm{x}$ & $\mathrm{x}$ \\
\hline mushito & $\mathrm{x}$ & $\mathrm{x}$ \\
\hline nal-tel & $\mathrm{x}$ & $\mathrm{x}$ \\
\hline olotillo & $\mathrm{x}$ & $\mathrm{x}$ \\
\hline tabloncillo & $\mathrm{x}$ & \\
\hline tepecintle & $\mathrm{x}$ & $\mathrm{x}$ \\
\hline tuxpeño & $\mathrm{x}$ & $\mathrm{x}$ \\
\hline vandeño & & $\mathrm{x}$ \\
\hline zapalote grande & $\mathrm{x}$ & $\mathrm{x}$ \\
\hline
\end{tabular}

Brush \& Perales, 2007). La variación intraracial exhibió patrones complejos: tuxpeño, tepecintle, y olotillo estuvieron conformados por muchas variedades tradicionales, mientras que comiteco y tabloncillo solo por una (tabla 4). Esta variación no está relacionada con el rango de distribución de la raza. Por ejemplo, zapalote grande tiene sólo una cuarta parte de las variedades tradicionales en comparación con olotillo en el mismo intervalo altitudinal de distribución.

Algunas razas están distribuidas a lo largo del intervalo altitudinal, como olotillo, tepecintle o tuxpeño, las cuáles de hecho son las más ampliamente distribuidas en la zona de estudio. Por el contrario, algunas crecen sólo en partes altas (comiteco), medias (vandeño y nal-tel) o bajas (conejo y tabloncillo) (fig. $5)$ y son cultivadas por pocos agricultores. La distribución de las razas entre las co- munidades indica que la mayoría de los agricultores siembran entre tres y cinco. El comiteco es la única raza que se siembra sola, lo que ocurre en las altitudes mayores de la zona de estudio (únicamente en Juquilita). Algunas razas crecen sólo en dos comunidades, como nal-tel que crece en Magdalena y San Francisco Loxicha, ambas comunidades ubicadas en las partes medias y bajas de la zona de estudio (fig. 6).

La distribución de las razas entre las comunidades indica que San Agustín Loxicha concentra el mayor número de ellas. Sin embargo, no existe una relación entre el tamaño del municipio y el número de razas cultivadas. Algunas razas se siembran únicamente en un municipio, como comiteco que sólo se siembra en San Agustín Loxicha y tabloncillo en San Baltazar Loxicha. Algunas están distribuidas sólo en dos mu- 
Tabla 4. Número de variedades tradicionales (VT) que comprenden cada una de las 10 razas registradas en el presente estudio.

Se indica el rango altitudinal en el que se distribuye cada una.

\begin{tabular}{lcccc}
\hline \multicolumn{1}{c}{ Raza } & Mínima & Máxima & Variedades & Intervalo \\
\hline comiteco & 1881 & 1881 & 1 & 0 \\
conejo & 264 & 1046 & 1 & 782 \\
mushito & 259 & 2114 & 6 & 1855 \\
nal-tel & 442 & 1065 & 2 & 623 \\
olotillo & 173 & 2094 & 14 & 1921 \\
tabloncillo & 1500 & 1500 & 1 & 0 \\
tepecintle & 173 & 2114 & 10 & 1941 \\
tuxpeño & 144 & 2094 & 12 & 1950 \\
vandeño & 314 & 2031 & 2 & 1717 \\
zapalote grande & 173 & 1628 & 4 & 1455 \\
\hline
\end{tabular}

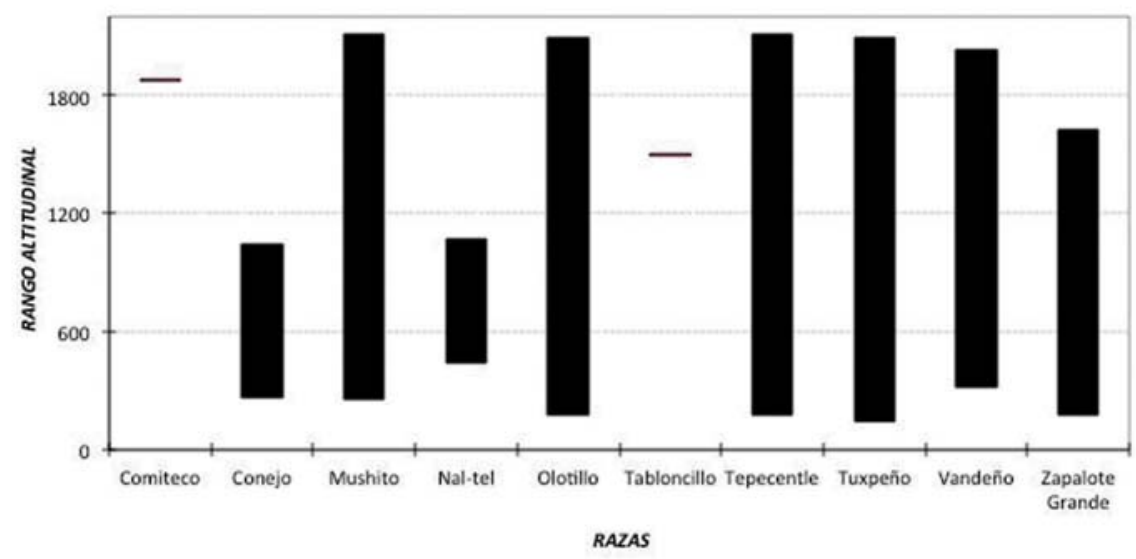

Fig. 5. Distribución altitudinal de las razas agronómicas en el sitio de estudio. 


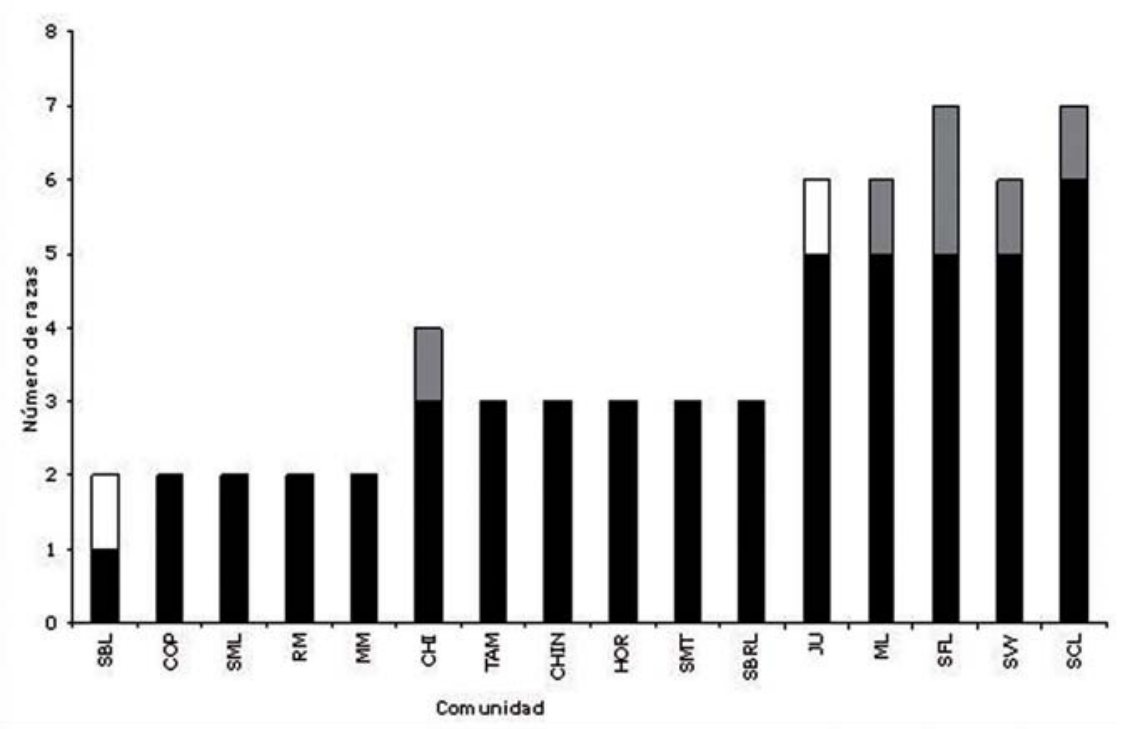

Fig. 6. Distribución de las razas entre las comunidades muestreadas: número de razas totales (negro); número de razas únicas (blanco); razas encontradas en dos comunidades (gris). (SBL: San Baltazar Loxicha; $\mathrm{COP}=$ Copalita; $\mathrm{SML}=$ Santa Martha Loxicha; $\mathrm{RM}=$ Río Molino; $\mathrm{MM}=$ Miramar $; \mathrm{CHI}=$ El Chilar; TAM = El Tamarindo; CHIN = La Chinilla; HOR: Los Horcones; SMT = Santa María Tepejipana; $\mathrm{SBRL}=$ San Bartolomé Loxicha; JU = Juquilita; $\mathrm{ML}=$ Santa María Magdalena Loxicha; $\mathrm{SFL}=$ San Francisco Loxicha; $\mathrm{SVY}=$ San Vicente Yogondoy; SCL = Santa Catarina Loxicha).

nicipios, como conejo que se encuentra en Candelaria y Santa Catrina Loxicha. Nal-tel se distribuye únicamente en San Agustín y San Bartolomé Loxicha. Vandeño sólo está presente en San Agustín y San Baltazar Loxicha (fig. 7).

Las 36 variedades tradicionales encontradas en este estudio apoyan los estudios previos que indican que la distribución de las razas de maíz es consecuencia de procesos complejos que involucran la selección humana y las condiciones ecológicas.

\section{Características agroecológicas de las razas}

A pesar de la gran variación existente dentro de cada raza y del alto porcentaje de sinonímia encontrado entre las variedades tradicionales, cuando se agrupan en sus respectivas razas las características de las mazorcas y semillas son estadísticamente diferentes, lo que indica que existen límites en su variación interna. Por ejemplo, tuxpeño, olotillo y zapalote grande exhibieron valores promedio altos en muchas características (tabla 5) y fueron estadísticamente diferentes de 


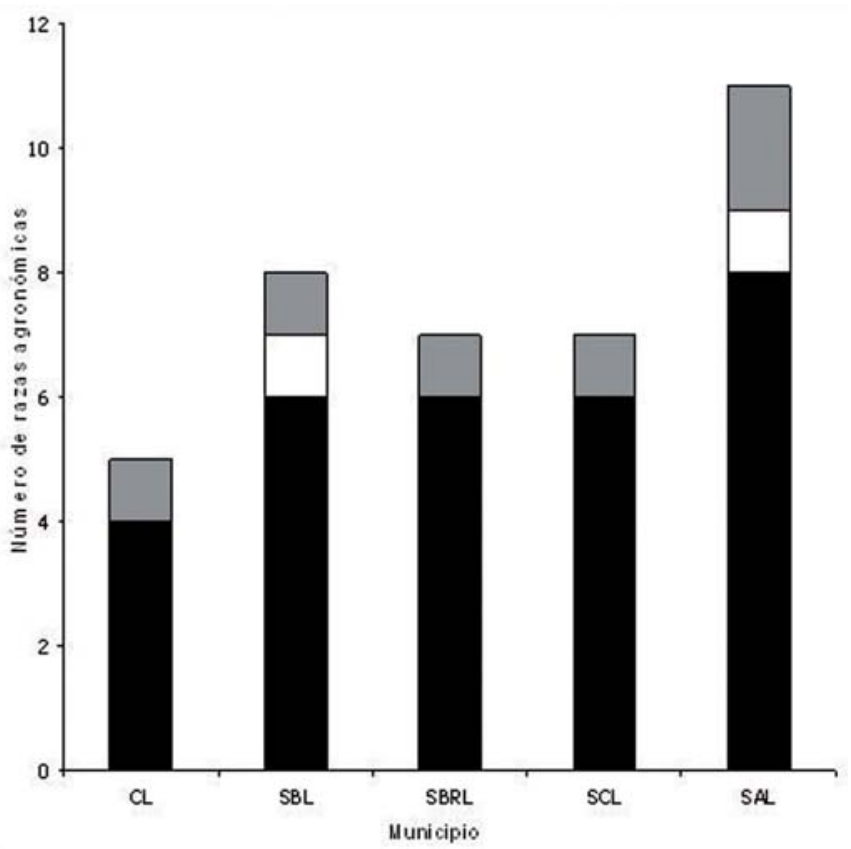

Fig. 7. Distribución de las razas agronómicas entre los cinco municipios estudiados: número de razas totales (negro); número de razas únicas (blanco); número de razas que se comparten en dos municipios (gris). (CL= Candelaria Loxicha; $\mathrm{SAL}=$ San Agustín Loxicha; $\mathrm{SBL}=\mathrm{San}$

Baltazar Loxicha; $\mathrm{SBRL}=$ San Bartolomé Loxicha; $\mathrm{SCL}=$ Santa Catarina Loxicha).

nal-tel o tepecintle, aún cuando comparten muchas variedades sinónimas.

Estos datos indican que cada raza ha sido seleccionada para diferentes características y aun cuando dos o tres variedades comparten el mismo nicho ecológico, cada raza mantiene su variación representativa.

Algunas razas que han sido reportadas previamente para un intervalo de altitud, en el presente estudio presentan variaciones (Ruiz-Corral et al., 2008). Esto indica que algunas de las razas presentan mayor adaptabilidad climática que otras, lo que es aprovechado por los agricultores para introducirlas a nuevos ambientes (Zeven, 1998; Ruiz-Corral et al., 2008).

\section{Manejo y selección de mazorcas y semillas}

De acuerdo con las encuestas, los agricultores obtienen semillas de diferentes fuentes:

1) Las semillas que provienen de la cosecha anterior. Los agricultores seleccionan las semillas más grandes y saludables (cuando el 


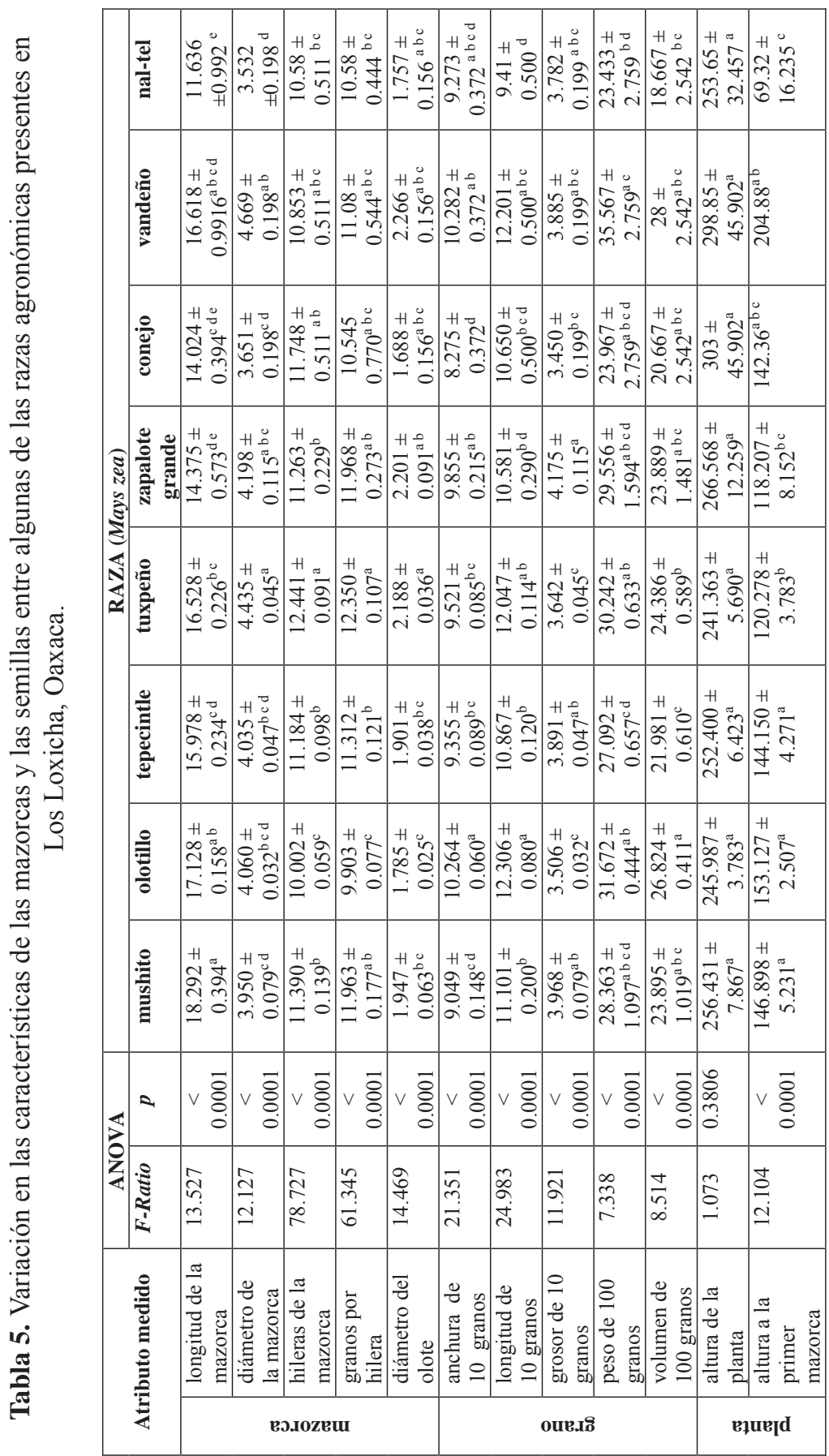


embrión tiene un color blanco y no presenta ningún tipo de daño). Los agricultores también separan las semillas de la parte media de la mazorca y la almacenan aparte.

2) Los agricultores obtienen semilla de otros agricultores, particularmente cuando pierden semilla del ciclo anterior debido a las malas cosechas, o cuando quieren probar otra variedad. Ellos también practican el "trueque", que ocurre cuando intercambian semillas de maíz por semillas de otras variedades, de otras especies o por otros productos (e.g. aceite, azúcar, flores).

3) Es común que los agricultores compren semilla fuera de la comunidad, en las tiendas especializadas en la venta de agroquímicos localizados en Pochutla, Ciudad de Oaxaca, Miahuatlán o diferentes lugares cerca de la costa (Puerto Escondido, Cozoltepec, Puerto Ángel, Santa María Tonameca, Santo Domingo).

La selección de semilla resume todos los criterios que los agricultores consideran necesarios para mantener una variedad tradicional como: tamaño de la mazorca y el olote, tamaño y peso de la semilla, altura de la planta, madurez y fenología, rendimiento, tolerancia a los vientos, sabor de las semillas, resistencia a los plaguicidas, textura y dureza de las tortillas, resistencia al daño, asignación biomasa vegetativa/ biomasa reproductiva, resistencia a altas o bajas temperaturas. Por ejemplo, variedades de ciclo corto son preferidas en aquellas áreas donde los vientos son comunes. En áreas templadas se prefieren las variedades de días largos. El manejo tradicional de la semilla es similar al reportado en estudios previos (Bellon \& Brush, 1994; Almekinders et al., 1994; Louette \& Smale,
2000) y representa la base del manejo de la diversidad morfológica y genética. Un manejo dinámico de los tipos de semilla en el espacio y el tiempo involucra el mantenimiento de semillas producidas localmente que satisfagan las necesidades ecológicas y culturales (Bellon, 2004). La pérdida de las semillas locales generalmente ocurre cuando hay reemplazamiento por otras que aparentemente responden a dichas condiciones y ocurre lo contrario. Sin embargo, la dinámica de las semillas locales es muy compleja. Ocasionalmente hay un reemplazo de variedades locales, de manera que variedades que llevan sembrándose varios años, al siguiente ciclo ya son reemplazadas por la mayoría de los agricultores, ya sea por variedades antiguas o por novedades que hayan surgido. El argumento es que esas variedades ya se han "cansado" y necesitan ser sustituidas por otras. Este fenómeno ha sido reportado previamente (Bellon \& Brush, 1994; Louette \& Smile, 2000; Perales et al., 2003; Bellon, 2004). La pérdida parcial de las semillas locales ocurre cuando se introducen variedades mejoradas las cuales tienen eventos de introgresión y se forman variedades criollizadas, por lo que las variedades locales adquieren ciertas características de las mejoradas y viceversa (Bellon \& Brush, 1994; Bellon \& Risopoulus, 2001; Louette et al., 1997; Louette \& Smale, 2000; Perales et al., 2003).

Todas aquellas variantes en el manejo de las semillas constituyen fuentes potenciales de ingreso de alelos a las variedades locales y que pueden ser fuente para la creación de nuevas variedades y así responder a las condiciones ambientales cambiantes o bien, para incrementar sus rendimientos (Bellon \& Taylor, 1993; Bellon \& Brush, 1994; Cleveland et al., 1994). 


\section{DISCUSIÓN}

Comparada con algunos estudios desarrollados en diferentes estados de México, y aún dentro del mismo estado de Oaxaca, la diversidad reportada en el presente estudio es alta en términos de las variedades tradicionales y razas agronómicas mencionadas. Estudios previos realizados en diferentes comunidades en los Valles Centrales de Oaxaca (Smale et al., 1999; Bellon et al., 2006; Bellon, 2004) y aquellos desarrollados en la región mixteca (Chávez-Servia et al., 2011; Soriano et al., 2011; Vera-Guzmán, 2012) mencionan menor diversidad. La distribución de las 36 variedades tradicionales encontrada en el presente estudio apoya estudios previos que indican una compleja interacción entre las condiciones ecológicas y la selección humana.

La interacción genotipo-ambiente favorece que las variedades tradicionales pertenecientes a diferentes razas agronómicas respondan a las mismas condiciones ecológicas, permitiendo la coexistencia de razas simpátricas (Brush \& Perales, 2007). Este efecto puede ser ilustrado por la distribución de las razas olotillo, tuxpeño y tepecintle que coexisten en el mismo gradiente altitudinal. Esta coexistencia también está relacionada con su origen e historia particular. Olotillo es una raza que crece en diferentes ambientes de la Depresión Central de Chiapas (Bellon $\&$ Brush, 1994). En Oaxaca también crece en muchas partes. Por su parte, tuxpeño está ampliamente distribuida en todo el mundo y es altamente productiva. Al parecer, fue domesticada en la región de OaxacaChiapas y ha sido utilizada en programas de mejoramiento de algunas variedades y para la generación de híbridos (Brush \& Perales, 2007). La distribución altitudinal registrada para esta raza en el presente estudio corresponde a aquella reportada por Ruiz-Corral et al., (2008). La excepción fue observada con tepecintle, porque los mismos autores reportan su distribución de los 0 a los 1400 m.s.n.m, mientras que en el presente estudio fue encontrada arriba de los 1800 m.s.n.m.

La adaptación local de algunas razas a ambientes específicos origina ecotipos. En este caso se encontró a la raza comiteco, la cual es originaria de las partes altas de Chiapas y su distribución es limitada (Brush \& Perales, 2007). En este estudio se encontró que también crece arriba de los 1800 m.s.n.m. y está representada por una sola variedad tradicional (blanco grande).

Finalmente, algunas razas pueden "moverse" fuera de su área original de distribución vía las variedades tradicionales que los agricultores van generando y que tienen el potencial de responder a nuevas condiciones ambientales.

Algunas razas que han sido reportadas sólo para las partes medias, como el tepecintle o el zapalote grande, en el presente estudio fueron encontradas fuera del área de distribución reportada previamente por Ruiz-Corral et al. (2008) en una publicación que resume los datos obtenidos durante 34 años por el Latin American Project. Esto sugiere que algunas razas mantienen su hábitat específico y que están adaptadas a dichas condiciones, mientras otras razas tienen mayor movilidad y muestran mayor plasticidad fenotípica y/o mayor diversidad genética y, por lo tanto, pueden ser utilizadas por los agricultores para cultivarlas en ambientes nuevos (Zeven, 1998; Ruiz-Corral et al., 2008). La variación fenotípica de 
las razas resume el papel social, cultural y económico que pueden jugar dentro de las comunidades, además del componente ecológico (Brush, 1995; Brush et al., 1992; Ferguson \& Mkandawire, 1993; Longley \& Richards, 1993; Soleri \& Cleveland, 1993; Cleveland et al., 1994). Si algunas razas exhiben altos niveles de variación fenotípica, más variedades tradicionales se pueden seleccionar y más ambientes podrían ser cultivados con ellas (Brush \& Perales, 2007). Aun cuando la selección humana y la deriva génica reducen el acervo genético de las variedades tradicionales, el manejo tradicional y los eventos de introgresión entre las razas pueden aumentar la diversidad genética y en consecuencia, su respuesta a las diferentes condiciones ecológicas. Éste es el caso del tuxpeño, olotillo y tepecintle, los cuales exhiben altos niveles de introgresión y tienen un gran número de variedades tradicionales. Este flujo génico entre las razas ha sido reportado previamente, indicando que los complejos raciales son muy comunes en las poblaciones de maíz con manejo tradicional (Chávez-Servia et al., 2011).

\section{CONCLUSIONES}

La alta diversidad de maíz en la Sierra Sur de Oaxaca resume un largo proceso de selección humana empírica y de adaptación local a un gradiente altitudinal. Los zapotecos han seleccionado diferentes variedades tradicionales para cubrir sus necesidades agronómicas, pero también culturales. En este proceso, la diversidad local ha sido preservada, lo cual también se observa en la adición de nuevos registros a la diversidad de maíz reportada para esta zona y para todo el estado de Oaxaca (CONABIO, 2011).
La presencia de un gran número de variedades locales apoya los resultados obtenidos previamente que indican que el maíz es el principal cultivo en las zonas rurales de México y por lo tanto, los agricultores continúan seleccionando y creando nuevas variedades locales. El manejo tradicional de la semilla es la base para el flujo génico entre poblaciones (en este caso, entre variedades y razas), la preservación y la introducción de alelos nuevos, lo cual contribuye a la diversidad genética del maíz.

Esta diversificación es intensificada debido a la introducción de razas foráneas (e.g. comiteco o tuxpeño) que incrementaron la diversidad genética y fenotípica existente. Los estudios sobre diversidad de maíz en México son necesarios para documentar la dinámica espacial y temporal de dicha diversidad, los cambios relacionados con procesos naturales y antrópicos y para promover la transferencia de tecnología basada en variedades locales.

\section{AGRADECIMIEnTos}

Agradecemos a la Comisión Nacional para el Conocimiento y Uso de la Biodiversidad (CONABIO) por el financiamiento del proyecto FZ003 "Diversidad y distribución altitudinal de maíces nativos en la Región de los Loxicha, Sierra Madre del Sur, Oaxaca". Al doctor Rafael Ortega-Paczka, quien identificó el material recolectado. Al doctor Jesús Cuevas Axayácatl por recibir el material e incorporarlo al Banco Nacional de Maíz, ubicado en Chapingo, Estado de México. A la Universidad Autónoma Metropolitana unidad Iztapalapa por el apoyo logístico y administrativo durante el desarrollo del mismo. 


\section{LITERATURA CITADA}

Aguilar, J., C. Illsley, y C. Marielle, 2003. "Los sistemas agrícolas de maíz y sus procesos técnicos". Esteva, C., y Marielle, C. (Eds.). Sin maíz no hay país. Consejo Nacional para la Cultura y las Artes. Dirección General de Culturas Populares e Indígenas, México, pp. 123-154.

Almekinders, C.J.M., N.P. Louwaars, y G.H. de Bruijn, 1994. "Local seed systems and their importance for an improved seed supply in developing countries". Euphytica, 78: 207-216.

Altieri, M.A., 2004. "Aspectos socio-culturales de la diversidad del maíz nativo". Maíz y biodiversidad: efectos del maíz transgénico en México. Conclusiones y recomendaciones. Informe del Secretariado de la Comisión para la Cooperación Ambiental, pp. 9.

Álvarez-Buylla, E., 2004. “Aspectos ecológicos, biológicos y de agrobiodiversidad de los impactos del maíz transgénico". Maiz y biodiversidad: efectos del maíz transgénico en México. Conclusiones y recomendaciones. Informe del Secretariado de la Comisión para la Cooperación Ambiental, pp. 24.

Aragón-Cuevas, F., F.H. Castro, E. Paredes, N. Dillánes, J.M. Hernández, S. Taba, y J. Díaz, 2002. "In situ conservation and participatory breeding of milpa in Oaxaca". Memorias del Simposio Manejo de la diversidad cultivada en los agroecosistemas tradicionales. IPGRIFuture Harvest-SDC. pp. 124-130.
Aragón-Cuevas, F., E. Paredes, H. Castro, S. Taba, y J. Díaz, 2000. "Conservation in situ and improvement of milpas in the Sierra Norte of Oaxaca, México". Abstract. Scientific basis of participatory plant breeding and conservation of genetic resources. Oaxtepec, Morelos.

Aragón-Cuevas, F., S. Taba, G.H. Castro, C.J.M. Hernández, T.J.M. Cabrera, A.L. Osorio, y R.N. Dillánes, 2003. "In situ Conservation and Use of Local Maize Races in Oaxaca, México: A participatory and decentralized Approach". Taba, S. (Ed.) Latin American Maize Germoplasm Conservation: Regeneration, In situ Conservation, Core subsets, and Prebreeding. Proceedings of a Workshop held at CIMMYT.

Aragón-Cuevas, F.; S. Taba, J.M. Hernández, J.D. Figueroa, V. Serrano, 2006. "Actualización de la información sobre los maíces criollos de Oaxaca". Instituto Nacional de Investigaciones Forestales Agrícolas y Pecuarias, Informe final SNIB-CONABIO proyecto No. CS002 México DF.

Arriaga, L., J.M. Espinoza, C. Aguilar, E. Martínez, L. Gómez, y E. Loa, 2000. Regiones Terrestres Prioritarias de México. CONABIO.

Badstue, L.B., R.M. Bellon, X. Juárez, R.I. Manuel, y A.M. Solano, 2002. "Social relations and seed transactions among small-scale maize peasants' access to maize landraces in the Central Valleys of Oaxaca, México, CIMMYT". Economics Working Paper 02-02. México, DF 28 pp. 
$\underline{\text { Rendón Aguilar, B. et al.: Diversidad de maíz en la Sierra Sur de Oaxaca, México: conocimiento y manejo tradicional }}$

Bellon, M.R., 2004. "Conceptualizing interventions to support on-farm genetic resource conservation". World Development, 32: 59-172.

Bellon, M.R., y J. Berthaud, 2006. "Traditional Mexican agricultural systems and the potential impacts of transgenic varieties on maize diversity". Agriculture and Human Values, 23: 3-14.

Bellon, M.R., y S.B. Brush, 1994. "Keepers of maize in Chiapas, Mexico". Economic Botany, 48: 196-209.

Bellon, M.R., y J. Risopoulos, 2001. "Small-scale farmers expand the benefits of improved maize germplasm: A case study from Chiapas, Mexico". World Development, 29: 799-811.

Bellon, M.R., y J.E. Taylor, 1993. "“Folk' soil taxonomy and the pamal adoption of new seed varieties". Economic Development and Cultural Change, 41: 763-786.

Brush, S., J.E. Taylor, y M.R. Bellon, 1992. "Technology adoption and biological diversity in Andean potato agriculture". Journal of Development Economics, 39: 365-387.

Brush, S.B., 1995. "In situ conservation of landraces in centers of crop diversity". Crop Science, 35: 346-354.

Brush, S.B., y H.R. Perales, 2007. “A maize landscape: Ethnicity and agro-biodiversity in Chiapas Mexico". Agriculture, Ecosystems and Environment, 121: $211-221$.
Chávez-Servia, J.L.; P. Diego-Flores, y J.C. Carrillo-Rodríguez, 2011. "Complejos raciales de poblaciones de maíz evaluadas en San Martín Huamelulpan, Oaxaca". Ra Ximhai, 7: 107-115.

Cleveland, D.A., D. Soleri, y S.E. Smith, 1994. "Do Folk Crop Varieties Have a Role in Sustainable Agriculture?" BioScience, 44: 740-751.

Comisión Nacional para el Conocimiento y Uso de la Biodiversidad. 2011. www. biodiversdidad.gob.mx/genes/proyectoMaices.html

Doebley, J.F., y M.M. Goodman, 1984. "Isoenzymatic Variation in Zea (Gramineae)". Sistematic Botany, 9: 203218

Dyer, G.A., y A. Yúnez-Naude, 2003. "NAFTA and conservation of maize diversity in México". Memories of The Second North American Symposium on Assesing the Environmental Effects of Trade. Comisión for Environmental Cooperation.

Esteva, G.R., 2003. "Los árboles de las culturas mexicanas". Esteva, C., y C. Marielle (Eds.). Sin maíz no hay país. Consejo Nacional para la Cultura y las Artes, Dirección General de Culturas Populares e Indígenas, México, pp. 17-28.

Ferguson, A.E., y R.M. Mkandawire, 1993. "Common beans and farmer managed diversity: Regional variations in Malawi". Culture and Agriculture Bulletin, 45-46: 14-17. 
Hernández-Xolocotzi, E., y R. Ortega, 1973. Variación en maíz y cambios socioeconómicos en Chiapas, México. 1946-1971. Avances en la Enseñanza y la Investigación en el Colegio de Posgraduados, pp. 11-12.

Herrera-Cabrera, E.B.; F. Castillo-González, J.J. Sánchez-González, J.M. Hernández-Casillas, R.A. Ortega-Paczka, y M. Major-Goodman, 2004. "Diversidad del Maíz Chalqueño". Agrociencia, 38: 191-206.

Instituto Nacional de Estadística Geografía e Informática, 2000. Censo de población y vivienda 2000.

Instituto Nacional de Estadística y Geografía, 2011. Anuario de estadísticas por entidad federativa 2011.

Longley, C., y P. Richards, 1993. "Selection strategies of rice farmers in Sierra Leone". De Boef, W., Amanor, K., Wellard, K., Bebbington, A. (Eds.). Cultivating Knowledge: Genetic Diversity, Farmer Experimentation and Crop Research. Intermediate Technology Publications, London, UK, pp. 51-57.

Louette, D.; A. Charrier, y J. Berthaud, 1997. "In situ conservation of maize in Mexico: Genetic diversity and maize seed management in a traditional community”. Economic Botany, 51: 20-38.

Louette, D., y M. Smale, 2000. "Farmers' seed selection practices and traditional maize varieties in Cuzalapa, Mexico". Euphytica, 113: 25-41.
Luna-José, A.L., y B. Rendón, 2008. "Recursos vegetales útiles en diez comunidades de la Sierra Madre del Sur, Oaxaca, México". Polibotánica, 26: $193-242$.

Luna-José, A.L., 2006. "Cuantificación de los recursos vegetales recolectados en tres municipios zapotecos de la Sierra Madre del Sur, Oaxaca". Tesis de maestría. Colegio de Posgraduados, Montecillos, México.

Matsuoka, Y.; Y. Vigouroux, M.M. Goodman, G.J. Sánchez, E. Buckler, y J. Doebley, 2002. "A single domestication for maize shown by multilocus microsatellite genotyping". Proceedings of the National Academy of Sciences of the United States of America, 99: 6080-6084.

Montalvo, L., 2006. "Manejo y estructura de la vegetación leñosa de los cafetales en la Sierra Madre del Sur, Oaxaca". Tesis de maestría. Colegio de Posgraduados, Montecillos, México.

Ortega-Paczka, R., 2003. "La diversidad del maíz en México". Esteva, C., Marielle, C. (Eds.). Sin maíz no hay país. Consejo Nacional para la Cultura y las Artes, Dirección General de Culturas Populares e Indígenas, México, pp. 123-154.

Ortiz-García, S.; E. Ezcurra, B. Schoel, F. Acevedo, J. Soberón, y A.A. Snow, 2005. "Abscence of detectable transgenes in local landraces of maize in Oaxaca, Mexico (2003-2004)". Proceedings of the National Academy of Sciences, 102: 12338-12343. 
$\underline{\text { Rendón Aguilar, B. et al.: Diversidad de maíz en la Sierra Sur de Oaxaca, México: conocimiento y manejo tradicional }}$

Ortiz-García, S.; E. Ezcurra, B. Schoel, F. Acevedo, J. Soberón, y A.A. Snow, 2006. "Transgenic maize in Mexico". BioScience, 56: 709.

Perales, R.H.; S.B. Brush, y C.O. Qualset, 2003. "Landraces of maize in Central Mexico: an altitudinal Transect". Economic Botany, 57(1): 7-20.

Piperno, D.R., y K.V. Flannery, 2001. “The earliest archeological maize (Zea mays L.) from highland Mexico: new accelerator mass spectrometry dates and their implications". Proceedings of National Academy of Sciences, 98 : 2101-2103.

Pressoir, G., y Berthaud, 2004a. "Patterns of population structure in maize Central Valleys of Oaxaca in Mexico". Heredity, 92: 88-94.

2004b. "Population structure and strong divergent selection shape phenotypic diversification in maize landraces". Heredity, 92: 95-104.

Ruíz, J.A.; J.J. Sánchez, y S.M. Aguilar, 2001. "Potential geographical distribution of teosinte in México: a GIS approach". Maydica, 46: 105-110.

Ruíz-Corral, J.A., N. Duran, J.J. Sánchez, J. Ron, D.R. Gonzalez, J.B. Holland, y G. Medina, 2008. "Climatic adaptation and ecological descriptors of 42 mexican maize races". Crop Science, 48: 1502-1512.

Sánchez, G.J.J., M.M. Goodman, y C.M. Stuber, 2000. "Isozymatic and Mor- phological diversity in the races of maize of Mexico". Economic Botany, 54: 43-59.

Sánchez, G.J.J., y A. Ruíz, 1996. "Distribución del teosinte en México". Serratos, J.A., Wilcox, C.A., Castillo, F. (Eds.). Flujo genético entre maíz criollo, maíz. mejorado y teosinte: implicaciones para el maíz transgénico. CIMMYT Economics Working Paper México, pp, 20-38.

Smale, M.; A. Aguirre, M. Bellon, J. Mendoza, e I.R. Manuel, 1999. Farmer management of maize diversity in the Central Valleys of Oaxaca, México. CIMMYT Economics Working Paper México, DF: CIMMYT 99-09, 27 pp.

Smith, B.D., 2001. "Documenting plant domestication: The consilience of biological and archaelogical approaches". Proceedings of the Natural Academy of Sciences, 98: 1324-1326.

Soleri, D., y D.A. Cleveland, 1993. "Hopi crop diversity and change". Journal of Ethnobiology, 13: 203-231.

Soleri, D.; D.A. Cleveland, y F. AragónCuevas, 2006. "Transgenic crops and crop varietal diversity: The case of maize in Mexico". BioScience, 56: 503-513.

Soleri, D., S.E. Smith, 2002. "Rapid estimation of broad sense heritability of farmer-managed maize populations in the Central Valleys of Oaxaca, Mexico, and implications for improvement". Euphytica, 128: 105-119. 
Soriano, R.; L. Arias, O. Bonilla, y J. Haro, 2011. "Programa de desarrollo comunitario agropecuario y ambiental participativo en una comunidad mixteca: Cosoltepec, Oaxaca". Desarrollo, Ambiente y Cultura, 1: 5-13.

Ventura-Aquino, Y.; B. Rendón, S. Rebollar, y G. Hernandez, 2008. "Use and conservation of forest resources in the municipality of San Agustin Loxicha, Sierra Madre del Sur, Oaxaca, Mexico". Agroforestry Systems, 73: 167-180.

Vera-Guzmán, A.M.; J.L. Chávez-Servia, y J.C. Carrillo-Rodríguez, 2012. "Proteína, lisina y triptófano en poblaciones nativas de maíz mixteco". Revista Fitotecnia Mexicana, 35: 7-13.
Wellhausen, E.; J. Roberts, L.M. Roberts, y E. Hernández, 1952. Races of Maize in Mexico: Their Origin, Characteristics, and Distribution. Harvard University Press, Cambridge, MA. 223 pp.

Wilkes, G.H., 1977. "Hybridization of maize and teosinte, in Mexico and Guatemala and the improvement of maize". Economic Botany, 31: 254-293.

1986. "Teosinte in Oaxaca, Mexico". Maize genet. Crop. News, 60: $29-30$.

Zeven, A.C., 1998. "Landraces: A review of definitions and classifications". Euphytica, 104: 127-139. 